\title{
Enhanced Luminescence Performance by Controlling the Er and Yb Distribution in the Nanometer Scale
}

\author{
A.Suarez-Garcia, R. Serna, M. Jiménez de Castro, C. N. Afonso, I. Vickridge* \\ Instituto de Optica, CSIC, Serrano 121, 28006, Madrid, Spain, *GPS, Universites Paris VI et VII, UMR 7588, Tour 23 \\ 2, Place Jussieu, 75251-Paris Cedex 5, France
}

Er doped fibers are currently used as amplifiers at $1.53 \mu \mathrm{m}$ in long distance communications for which several hundred meters of Er-doped fibers are used. However, in scaling down the device dimensions to substitute the fiber amplifier by an integrated one, the Er concentration has to be increased to achieve enough optical gain. Physical processes related to energy transfer and luminescence efficiency become then a key issue in integrated amplifiers. For practical applications another important factor that limits the Er doped amplifier performance is the small absorption cross-section of Er at $980 \mathrm{~nm}$. Instead, Yb has an absorption cross-section extended over a wide spectral range (850$1000 \mathrm{~nm}$ ) that is about one order of magnitude higher than that of Er at this wavelength. It is well known that Yb can transfer resonantly the energy to Er upon de-excitation, the efficient energy transfer of the energy being essential to achieve an optimum luminescent response. We have recently produced Er: $\mathrm{Al}_{2} \mathrm{O}_{3}$ films with Er concentrations higher than $10^{20}$ ions $/ \mathrm{cm}^{3}$ and an improved luminescence response, [1] by controlling the Er ion distribution in the nanometric scale.

The aim of this work is to show how the Er-Yb transfer can be optimised by controlling not only the Er and $\mathrm{Yb}$ relative concentrations, but also by nanostructuring the rare earth ion distribution to the nanometric scale. The films are grown by alternate deposition of amorphous aluminum oxide $\left(\mathrm{Al}_{2} \mathrm{O}_{3}\right)$, Er and $\mathrm{Yb}$ layers. The Er-Er ion layer separation

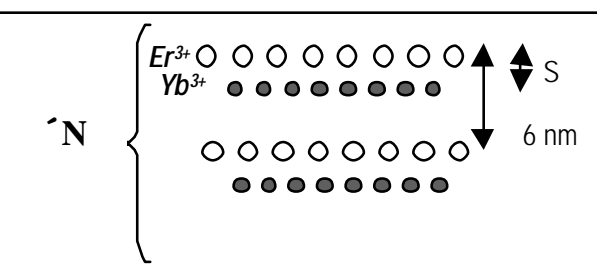

Fig. 1. Schematic diagram of the $\mathrm{Er}$ and $\mathrm{Yb}$ ions within the $\mathrm{Al}_{2} \mathrm{O}_{3}$ host. $\mathrm{N}=25$ and $\mathrm{S}$ varies from 0 tc $3 \mathrm{~nm}$. was fixed to $6 \mathrm{~nm}$ since in an earlier work we found that separations equal or higher than this value gave the optimum luminescence performance. [1] Layers of $\mathrm{Yb}$ ions are produced for each Er layer, so layers of ions of different nature were alternated (Fig. 1). The separation $S$ from Er to $\mathrm{Yb}$ layers was varied from 0 (Er and $\mathrm{Yb}$ ions together in the same layer) to $3 \mathrm{~nm}$ ( $\mathrm{Yb}$ layers centered within two $\mathrm{Er}$ layers). We have synthesized $0.3 \mu \mathrm{m}$ thick thin films of amorphous $\mathrm{Al}_{2} \mathrm{O}_{3}$ doped with a constant $\mathrm{Er}$ content of $6 \times 10^{15} \mathrm{at} \cdot \mathrm{cm}^{-2}$ and a relative $\mathrm{Er}: \mathrm{Yb}$ content variable from 1:0 (non codoped film as a reference) to 1:4.

The photoluminescence (PL) of Er at $1.53 \mu \mathrm{m}$ was pumped by a Ti-sapphire laser in the 910-990 spectral range and shows that the the $1.5 \mu \mathrm{m}$ emission in the Er doped films (with no $\mathrm{Yb}$ doping) can only be excited in a very narrow band (FWHM 10 nm) around $975 \mathrm{nn}$. The PL emission in the Er-Yb co-doped films was observed by pumping at any wavelength in the studied range thus evidencing the existence of energy transfer from Er to Yb. PL measurements performed using a fixed pump wavelength at $975 \mathrm{~nm}$ showed that the PL intensity was always higher in the Er-Yb codoped films than in the Er doped ones. It is also seen that the PL intensity increases as the Yb content is increased, thus the best luminescence response has been achieved for the film with an Er:Yb ratio of 1:4. Since no tendency to saturation is seen, it is most likely that higher $\mathrm{Yb}$ oncentrations could be used that would result in further improvement. Nevertheless it is remarkable that for any $\mathrm{Yb}$ concentration the optimum PL response results are obtained when the separation of the Er and $\mathrm{Yb}$ ion layers is equal to $1 \mathrm{~nm}$. The reasons why this separation optimizes the Er-Yb energy transfer and the foreseen optimized nanostructuring distribution will be finally discussed.

[1] R. Serna, M. Jiménez de Castro, J. A. Chaos, A. Suarez-Garcia, C. N. Afonso, M. Fernández, I. Vickridge. J. Appl. Phys., vol 90, No 10, pp 5120-5125 (2001). 